\title{
Contextos educativos inteligentes
}

\author{
Intelligent educational contexts
}

\author{
Daiana Yamila Rigo \\ Consejo Nacional de Investigaciones Científicas y Técnicas (CONICET) \\ Universidad Nacional de Río Cuarto (UNRC) \\ daianarigo@hotmail.com \\ Danilo Donolo \\ Consejo Nacional de Investigaciones Científicas y Técnicas (CONICET) \\ Universidad Nacional de Río Cuarto (UNRC) \\ donolo@hum.unrc.edu.ar
}

\section{Resumen}

Inteligencia y educación son dos constructos complejos. En el presente trabajo nos interrogamos acerca de ¿Cómo crear contextos educativos inteligentes? y ofrecemos algunas respuestas interesantes reconociendo las nuevas concepciones sobre inteligencia y su implicancia en educación. El escrito presenta desde un enfoque socio-constructivista las características que se le reconocen a los contextos educativos para ser pensados como inteligentes y el entramado de consideraciones que se realiza desde la psicología educacional y cultural para el desarrollo de nuevos enfoques de aprendizajes cuando se asume la importancia del contexto y del sujeto de aprendizaje. Asimismo, se describen las características que las tareas académicas y las evaluaciones adquieren cuando se busca construir contextos educativos inteligentes.

Palabras clave: educación, inteligencia, contexto, aprendizaje. 


\section{Abstract}

Intelligence and education are two complex constructs. In the present work we wonder about how to create smart educational contexts? and we offer some interesting answers, recognizing the new conceptions of intelligence and its implication on education. The paper presents a socio-constructivist approach of the characteristics which are recognized to educational contexts to be thought as intelligent and the lattice of considerations held from the cultural and educational psychology for the development of new learning approaches when it is assumed the importance of the context and the subject learning. Additionally, describes the features that academic tasks and evaluations acquire when looking to build intelligent educational contexts.

Key Words: education, intelligence, context, learning.

Fecha recepción: Septiembre 2013 Fecha aceptación: Octubre 2013

\section{Introducción}

\section{Cómo y dónde ocurre cognición y aprendizaje}

Generalmente se acepta que la inteligencia y el aprendizaje son cualidades o características propias de cada persona como individuo. Algunas teorías psicológicas han dado pie para mantener el supuesto, y así, cuando se habla de inteligencia la asociamos con un aspecto de la personalidad que nos ha sido 'regalada' en la concepción y se va a ir actualizado en aprendizajes a través de la vida.

Si bien como dijimos, la creencia está consolidada, algunos evidencias de estudios más recientes dan cuenta de una fuerte contribución social y ambiental tanto sobre la inteligencia y su desarrollo en los individuos como en los evidentes o sutiles matices que 
tiene el aprendizaje en distintos aspectos asociados con la vida así como en los aprendizajes académicos específicos de la escuela.

La iniciativa por mostrar el aspecto social y compartido en la configuración y desarrollo de inteligencia, cognición y aprendizaje es tentadora, por lo que tomaremos un espacio para delinear los aspectos que nos parecen más interesantes en la propuesta de las teorías educacionales y cómo ellas se pueden plasmar en experiencias instruccionales más eficientes y creativas.

En este sentido, el interés teórico-práctico por comprender el funcionamiento cognitivo inmerso en el contexto social, cultural e histórico se expresa en las investigaciones y los desarrollos teóricos que nutren el complejo entramado que implica aprender. Diversos autores parecen acordar, y entre ellos Alexander (2006), Perkins (1995), Rinaudo y Donolo (2000) y Rennie (2007) concentran sus estudios en algunas de las características que adquiere la construcción del conocimiento. Su análisis y descripción es objeto del presente escrito. Primero referimos al aprendizaje como un proceso idiosincrásico, luego describiremos al conocimiento como distribuido y situado, para finalizar con la importancia de considerar los contextos donde ocurre el conocimiento y el aprendizaje. La peculiaridad del conocimiento como algo idiosincrásico responde a Cómo aprenden las personas. Las diversas perspectivas que estudian el carácter singular del conocimiento y del aprendizaje acuerdan que son procesos personales que difieren en cada persona. Falk y Dierking (2000) refieren al contexto personal de aprendizaje para explicar los factores que median en la construcción del saber, refiriéndose a los motivadores intrínsecos y extrínsecos, a los intereses hacia tareas, a los programas o eventos determinados, a los conocimientos y experiencias previas y a las elecciones personales.

Respecto a las experiencias, los ambientes en que se desarrolla la vida diaria, muchas veces nos hace perder la perspectiva de lo particular de sus características y las diferencias con otros períodos históricos. La aparición de nuevas tecnologías hace suponer influencias 
marcadas en el pensar y en hacer. Si bien puede que su influencia esté escondida en lo cotidiano del vivir, a poco de pensar se nos hace palpable en las experiencias en las que se encuentran inmersos los niños y adolescentes de hoy, que avizoran una manera particular de interactuar con la realidad medida por la tecnología, Internet, juegos en red, youtube, wikipedias, que indudablemente marcan nuevas formas de ser inteligentes e intereses que deben atenderse en los contextos educativos. Respuestas que se están encontrando en los planteos de educación a distancia, el uso de modalidades de blended learning, los blog, las bibliotecas interactivas, los recursos educativos virtuales que permiten el desarrollo de capacidades que serán de relevancia para el futuro profesional de los jóvenes, aspecto que contrasta con generaciones anteriores donde el uso de capacidades más técnicas se presentaban como esenciales.

De acuerdo con las nuevas mediaciones que el contexto social ofrece se entiende que las lecturas que los sujetos realizan asumen un carácter diverso en la forma de interpretarlas e internalizarlas. En este sentido, Gardner (2006), Kelly y Tangney (2006) y Rigo y Donolo (en prensa) coinciden en destacar que los sujetos enfrentan la construcción de los saberes de maneras diversas, haciendo uso de distintas modalidades en sus procesos de adquisición y comprensión de los contenidos escolares, utilizando diversos recursos, en tiempo y espacios diversos.

Los planteos psico-educativos, también se inclinan a pensar que el conocimiento y el aprendizaje no se dan de manera solista. Los representantes y seguidores de las propuestas se interrogan Dónde está el conocimiento y cómo se construye, para aludir a la noción de cognición distribuida y situada. Desde esta perspectiva se entiende que el aprendizaje está socialmente contextualizado, es decir, que los significados que se construyen emergen de la interrelación que los sujetos mantienen con el contexto, que a su vez está mediada por herramientas, actividades, comunicaciones y sistemas simbólicos. En relación con la idea de conocimiento situado, el concepto de cultura cobra especial relevancia, destacándose que la actividad de aprender se desarrolla en un tiempo y en un 
espacio. En este sentido, Falk y Dierking (2000) mencionan la existencia de múltiples comunidades de aprendizaje, para especificar que el conocimiento no es el mismo en todos los individuos ni en todas las sociedades. Es importante notar que las experiencias que uno tiene la posibilidad de tomar más allá del contexto local marcan un camino de posibilidades para despertar pasión por determinadas actividades, fue durante sus vacaciones a África, que un joven de doce años -británico-, no sólo descubrió su pasión por la fotografía, si no que fue reconocido por Real Sociedad Fotográfica del Reino Unido, quien lo premió por las imágenes obtenidas ${ }^{1}$.

Este tipo de experiencias en contextos reales, nos animan a pensar no sólo al conocimiento como situado, si no también como distribuido. El término ha sido mencionado en la literatura con varias designaciones. Al respecto, Pea (1993) opta por estudiar la inteligencia distribuida, mientras que Perkins (1995) se refiere a cogniciones repartidas. Uno y otro para señalar, por un lado, que los conocimientos se construyen socialmente a través de esfuerzos cooperativos dirigidos a metas compartidas, diálogos o cuestionamientos que emergen de las distintas perspectivas consideradas por las personas. Y por otro lado, para sostener que la cognición y la inteligencia están física, social y simbólicamente repartidas.

Al respecto, Rinaudo y Donolo (2000) y Rinaudo (2007) reconocen que la inteligencia distribuida implica que las personas trabajen junto al entorno, en colaboración con otros sujetos, objetos físicos y simbólicos a través de los cuales se potencia las capacidades individuales. Los aportes de Alexander (2006) van en el mismo sentido, en tanto entiende que la responsabilidad cognitiva involucrada en cualquier tarea académica distribuida entre individuos promueve la comprensión y las habilidades de cada estudiante en una configuración que envuelve pensamiento colectivo, aprendizaje cooperativo e intercambios mentales.

1. 1 La nota fue publicada por el BBC el 3 de noviembre de 2011. El niño fotógrafo. Disponible en http://www.bbc.co.uk/mundo/video_fotos/2011/11/111101_galeria_fotos_nino_premio_az.shtml [consultado 03nov-2011]. 
Ambos aspecto, cognición situada y distribuida, han adquirido mayor relevancia con los años en los contextos educativos, más específicamente, la premisa de Perkins (1995) de la 'personas más el entorno' cobra valor en los contextos instruccionales. En este sentido, la participación de experiencias interculturales e intercambios estudiantiles da matiz a los aprendizaje de los sujetos en cuanto toman sentido de vivencia más cercana con la construcción del conocimiento, de igual modo, se presentan algunas iniciativas televisivas que invitan a conocer el lado cotidiano de la ciencia, como las conferencias en TEDx, Alterados por Pi de Paenza o Proyecto G de Golombek. Pero también nos encontramos con nuevos estilos de enseñar que haciendo junto a otros y en lugares no habituales promueven la comprensión de los contenidos curriculares, como la reciente iniciativa de trabajar con artistas en un museo, la integración de rock y el pop dentro del proyecto educativo tradicional ${ }^{2}$ o la idea de prácticas socio-comunitarias en los planes de estudio.

Con lo cual las experiencia señaladas, reflejan un tercer aspecto que busca comprender En qué contextos es factible aprender. El postulado entiende que el conocimiento refleja experiencias que ocurren tanto dentro como fuera del contexto escolar. En este sentido se trata de extender al entorno de aprendizaje más allá de las paredes de la clase, de hecho desde la Psicología Educacional se entiende que existe una multiplicidad de experiencias en el mundo real que pueden demostrar, reforzar o ampliar cualquier concepto que se desee enseñar (Alexander, 2006).

Entonces, cuando hablamos de contexto, lo hacemos en sentido amplio, al considerar no sólo el aula como entorno de aprendizaje, si no también otras comunidades de aprendizaje de carácter no formal, tales como los parques temáticos, los museos, los

\footnotetext{
${ }^{2}$ Ambas noticias, el trabajo de niños en museo junto a artísticas y la iniciativa de combinar la enseñanza de la música junto al bachillerato común, revelan innovaciones simbólicas que amplían los márgenes sobre dónde, cuándo y qué enseñar. Para más conocimiento: 1. Trabajo de artistas en el aula para que los niños expongan. LaNación online http://www.lanacion.com.ar/1403709-trabajo-de-artistas-en-el-aula-para-que-los-ninosexpongan?utm_source=newsletter\&utm_medium=titulares\&utm_campaign=NLCult [consultado 02-nov-2011]; 2. El rock y el pop, en una escuela muy particular. LaNación online http://www.lanacion.com.ar/1393725-el-rock-y-el-pop-enuna-escuela-muy-particular?utm_source=newsletter\&utm_medium=titulares\&utm_campaign=NLCult [consultado 02nov-2011].
} 
acuarios, las galerías de arte, las tertulias, entre otros. Espacios que nos ayudan a conocer mejor y saber más sobre los objetos culturales -arte rupestre, utensilios de caza, vestimenta típica-, sobre la flora y la fauna observando in situ, disfrutando de los aromas, las texturas y las variedades. Asimismo, los espacios y galerías de arte también han cobrado ímpetu en los últimos años con el surgimiento de nuevos museos que invitan a vivir el arte a través de lo estético, las emociones y sensaciones. Lo que se quiere decir es que se aprende mejor y se desarrollan otras habilidades si nos unimos a los recursos que nos facilitan el acceso al saber.

Por otro lado, considerando ambos contextos, se deriva dos concepciones de conocimiento. Un conocimiento informal o no escolarizado que da cuenta de una comprensión adquirida como resultado de una experiencia en un contexto no formal de educación, asociado a un aprendizaje incidental, y un conocimiento formal o escolarizado que se alcanza en experiencias educativas más estructuradas, que pone énfasis en la construcción de conceptos científicos a lo largo de un proceso de aprendizaje intencional.

El reconocimiento de tales características para el aprendizaje y la cognición, no sólo ameritan hablar de contextos inteligentes, en tanto las prácticas educativas que parten de las tres premisas delimitadas aportan a su definición, sino que, además, su consideración crea entornos que atienden a las individualidades de los estudiantes, las interrelaciones entendidas como construcciones mediadas por elementos físicos, sociales y simbólicos que toman sentido en un tiempo y en un espacio educativo, ya sea formal o no formal.

A la vez, desde una perspectiva práctico-pedagógica, entendemos que crear contextos educativos inteligentes es un desafío que integra promover proyectos educativos múltiples con sentido contextualizado y de carácter individual. Un ejemplo, se encuentran en la planificación de experiencia didácticas que consideran los múltiples perfiles intelectuales de los estudiantes, el abanico de sitios donde aprender y la importancia de reconocer los contextos y los artefactos que significan las acciones de los individuos. 


\section{Promover contextos inteligentes}

La perspectiva educativa y cultural apuesta, en sentido general, que el aprendizaje y la cognición se entienden en un marco que se define por ser idiosincrásico, situado y distribuido. Y a la vez, asumimos que los contextos educativos son inteligentes cuando se aproximan, entre otros aspectos, a los postulados que Bruner (1997) plantea cuando analiza la educación desde una perspectiva psico-educativa, axiomas que ayudan a pensar a los contextos como inteligentes en tanto actúan como guía para que alguien aprenda algo sobre otro.

Un primer aspecto refiere a la idea de perspectivismo, el cual puede ser pensado en dos sentidos. Por un lado, se entiende que el conocimiento y la educación se construyen desde múltiples entradas, no sólo desde la perspectiva del docente, si no que también se reconocen otros agentes que aportan de igual modo al hecho de aprender, como ser los alumnos, la familia, la comunidad de aprendizaje. Por otro lado, se comprende que las formas de conocer no se limitan a las modalidades canonizadas por la cultura, su amplitud se extiende a las expresiones artísticas, emocionales y académicas. Pareciera, que el enfoque ha cobrado vida en algunas escuelas que, para estimular el desarrollo de diversos talentos, conjugan el plan de estudio tradicional con la creación de entonos de instrucción personalizada para descubrir, explorar y potenciar a través de actividades contextualizadas y juegos que indagan acerca de las profesiones de los adultos -Escuela Sol en Bariloche, Argentina, The Gardner School of Arts \& Sciences en Vancouver, Washington, The Gardner School en Nashville, Tennessee, Multiple Intelligence International School en Quezon, Philippines y Key Learning Community en Indianápolis, Indiana-. O bien proyectos que diversos centros educativos ponen en marcha para estimular las inteligencias emocionales, tales como los aplicados en la Institución Educativa SEK en Barcelona, España o en los Centros Educativos Waldorf en Madrid, España. 
Un segundo aspecto se relaciona con la necesidad de ampliar los límites de lo que se comprende por pensar, conocer, sentir y percibir. Tal postulado reconoce la diversidad de sistemas simbólicos y dominios del saber que se observan tanto en los contextos educativos formales como no formales de educación. Desde una perspectiva práctica, el límite se asume sobre el tipo de conocimiento y sobre los contextos dominantes, pero desde una consideración pedagógica, saltar los límites envuelve el reconocimiento de múltiples saberes, inteligencias y entornos educativos.

Un tercer aspecto implica los modelos de enseñanza basados en la perspectiva de comunidades de aprendizaje, cuyo elemento clave es la tendencia a aprender trabajando juntos para mejorar la construcción social del conocimiento (Molina Ruiz, 2005). Como tal se muestra opuesto al enfoque encontrado en la mayoría de las escuelas, donde el aprendizaje es analizado como un proceso solista, cuya meta es trasmitir el conocimiento del docente o del libro de texto (Collins, 2006). En contraste, las comunidades de aprendizaje promueven el interés y los talentos individuales y en efecto, los aprendices se ayuden a aprender unos a otros, cada cual en función de sus habilidades. Es justamente en el seno de las comunidades donde emergen las alternativas para sentimos a gusto y apreciamos que podemos hacer lo que nos da placer, siendo más productivos, tal es el caso de Ferran Adrià, un chef que esta dando riendas sueltas a la cocina y la innovación en el plano de lo molecular, entrelazando la química, la física, el arte y la gastronomía. Son asimismo ejemplo de comunidades de aprendizaje los contextos extracurriculares, de ocio y de esparcimientos (talleres de arte, de tejido, de circo, malabarismo, jardinería, entre otros) que se gestan desde un enfoque de hacer y aprender junto a otros.

Un cuarto aspecto refiere a externalizar las obras y productos creados por los alumnos. Este postulado rescata la importancia de hacer público el conocimiento y de compartirlo a toda la comunidad educativa. El registro de los productos mentales se puede apreciar en diversa ocasiones: la maratón de lectura, la creación de un cuento, los concursos fotográficos, las ferias de ciencia, los talleres de pintura, entre otros. Que a la vez 
posibilitan la expresión de diversas inteligencias. No hace mucho, en un pueblo ubicado en el centro de Córdoba, Argentina, se desarrollaron la 8o Feria de Ciencia, con el motivo de exponer los trabajos realizados por los alumnos en ciencias sociales y naturales. La posibilidad de comunicar y exponer los productos y obras creadas muestran un clima distinto al de escolarización, los niños son los protagonistas activos, se muestran participativos y comprometidos en contar, mostrar y contestar a las preguntas del público. Tienen la posibilidad de desplegar habilidades interpersonales, convirtiéndose en una actividad contextualizada dirigida a una audiencia, y previamente de aplicar conocimientos de otras disciplinas como matemáticas, lengua e informática para guiar el proceso de investigación, formándose en destrezas que tendrán importancia en un contexto futuro de actuación.

Un quinto aspecto examina el poder de la cultura sobre la mente. No sólo se reconoce múltiples formas de usar la mente, de conocer y de interpretar al mundo, sino que es instrumental a distintas situaciones. El planteo se inclina a comprender la mediación que la cultura escolar facilita para el desarrollo de una u otras habilidades, la selectividad promovida con fines sociales. Sin embargo, el hecho de crear pequeña innovaciones simbióticas dentro o fuera de lo escolar o abrir espacios para su realización generan posibilidades de cultivar las múltiples capacidades de la mente como es planteado al interior de la teoría de las inteligencias múltiples (Gardner, 1983). En este sentido, en las escuelas se observan algunas ocasiones de innovar cuando participan de diversos tipos de olimpíadas, así como cuando se unen a las invitaciones para narrar historias, concurso de cortometrajes, la producción de contenidos educativos digitales o bien cuando se crean puentes entre diversas instituciones educativas como la experiencia llevada ya hace un tiempo entre una escuela secundaria y un laboratorio de ciencia, que invitaba a adolescentes de diecisiete años a hacer investigación ${ }^{3}$.

\footnotetext{
${ }^{3}$ La nota fue publicada por LaNación online el 22 de septiembre de 2000. Científicos precoses: de la escuela al laboratorio. Disponible en http://www.lanacion.com.ar/33877-cientificos-precoces-de-la-escuela-al-laboratorio [consultado 4-nov-2011].
} 
Un sexto aspecto, que integra a los restantes, se establece en la configuración de entornos educativos creativos, diversos y flexibles que amplían las posibilidades de expresión de talentos, formas de hacer y pensar de manera variada, potenciando múltiples modos de ser inteligentes.

Cada uno de estos postulados psico-educativos abre puertas para pensar una educación más allá de las formas canónicas que se observan día a día en las aulas, nos desafían a crear nuevos entornos más perspicaces. En este sentido, el apartado que sigue ofrece algunas pautas para cumplir con la meta planteada, crear contextos educativos inteligentes.

\section{Manos a la obra...qué implica crear contextos inteligentes}

De la comprensión de las tres características que definen al aprendizaje y la cognición y de la consideración de los seis postulados psico-educativos, se derivan algunas ideas clave para la educación en contextos inteligentes. Entendemos que si lo social deja huellas en la cognición, en el aprendizaje y en la inteligencia, y las experiencia que tomamos a diario se vuelven fuente de aprendizaje, la discusión acerca de la delimitación de las tareas académicas y las evaluaciones en su reconsideración hacia formulaciones educaciones más creativas, situadas y diversas es crucial. Presentamos algunas ideas en torno a dos ejes, tareas y evaluación en educación, con la finalidad de mostrar alternativas para generar entornos que apuesten a la diversidad, al carácter situado y distribuido de los aprendizajes y al desarrollo de habilidades.

Por un lado, respecto a las tareas académicas, consideramos que en su definición se juega la posibilidad de crear entornos que puedan motivar y comprometer a los estudiantes. Entre las características que se mencionan en la bibliografía específica sobre el tema se destacan que sean: diversas y variadas, auténticas, novedosas, abiertas, interesantes, complejas, que otorguen cierto nivel de autonomía en el planteo, realización y evaluación de la actividad y presenten un nivel de dificultad medio (Ames, 1992; Ames y Archer, 
1988; Doyle, 1983; Mitchell y Carbone, en prensa; Paoloni, 2010; Rosenholtz y Wilson, 1980; Stipek, 1996).

Estas dimensiones que adquieren las actividades académicas son reconocidas dentro de un enfoque socio-cognitivo y pensadas desde una perspectiva situada y distribuida. En lo referente al cómo diseñar e implementar múltiples tareas bajo esa perspectiva, el modelo de puertas de entrada de Gardner (2006) y Boix Mansilla (2004) es una alternativa innovadora y prometedora. En este sentido, es un modelo sugerente para llevar a la práctica los aspectos destacados sobre cognición y aprendizaje en los apartados anteriores, sustentándose en la teoría de las inteligencias múltiples para el diseño de experiencia pedagógicas.

Respecto a la diversidad y variedad en las tareas presentadas se ofrece un marco para trabajar sobre diferentes tipos de actividades, utilizando diversos recursos, materiales y herramientas. En este sentido, Gardner (2006) propone seis entry point para favorecer no sólo la comprensión de los contenidos curriculares, si no también promover orientación motivacional intrínseca y compromiso hacia los aprendizajes. El autor menciona las narraciones, la presentación de estructuras lógicas-argumentativas, el punto de vista existencial para fundamentar las facetas terminológicas de los conceptos, el enfoque estético para incluir el dominio artístico en el diseño de la clase, las experiencia vivénciales como visitas a museos, parques temáticos, entre otros y el trabajo en grupos colaborativos a través de proyectos, discusiones, debates y role-play para fomentar las relaciones interpersonales.

De este modo, la formulación de tareas académicas desde múltiples perspectivas abarca una variedad de representaciones, medios de información y sistemas simbólicos que ayudan a comprender el contenido de manera profunda, en tanto se requiere la puesta en marcha de diversos perfiles intelectuales, que a la vez dan cabida a la diversas habilidades de los estudiantes. 
El planteo de diversidad y de variedad de actividades, también se encuentra en consonancia con las tendencias actuales en investigación educativa, al respecto Alexander (2006) indica que las investigaciones que se están orientando dan a conocer en qué tareas los sujetos se muestran más inteligencias, y así mismo, definen qué estrategias de resolución utilizan para solucionar actividades o desarrollarse en un campo disciplinar determinado. Tal planteo invita a configurar contextos heterogéneos que brinden posibilidades de proponer tareas abiertas, tanto a nivel de formato como a nivel de productos, es decir, es el alumnos quien tiene la libertad de elegir qué clase de producto desarrollará frente a la amplitud de la tarea solicitada.

También los estudios en el campo educativo resaltan el carácter auténtico de las tareas académicas como promotor de aprendizajes más significativos. En lo referente, Perkins (1995) expresa que las prácticas pedagógicas hacia el aprendizaje situado en un contexto ofrecen una estructura y una motivación a los conocimientos y habilidades que se están aprendiendo. Mitchell y Carbone (en prensa) advierten que establecer relaciones con la vida cotidiana, presentando problemas que pongan en juego el uso y desarrollo de habilidades necesarias para el mundo cotidiano, fomenta no sólo el compromiso cognitivo sino también lo comportamental y lo afectivo.

Junto a la significatividad de las actividades diseñadas y el valor de utilizar variedad de representaciones en su formulación, se une la importancia de la novedad, la complejidad y el interés generado por las mismas. El propósito de incluir tales características en las actividades académicas está en mantener la motivación y la responsabilidad. Así, cuando se refiere a la novedad, no se alude al contenido, sino a la tarea en sí en cuanto forma de presentación y conforme las experiencias y conocimientos previos de los alumnos. Cuanto se atañe al grado de complejidad, se relaciona con el número de pasos que requiere la tarea, la cantidad de información a considerar y la cantidad de metas a alcanzar (Mitchell y Carbone, en prensa). Y cuando se puntualiza sobre el interés, Stipek (1996) sugiere que 
está determinado por el nivel de dificultad de la tarea, es decir, éstas serán percibidas como interesantes por los estudiantes cuando se presenten desafiantes a sus actuales estructuras cognitivas, o cuando desde la perspectiva vigotskiana se formulen dentro de la zona de desarrollo próximo.

Por último, Ames (1983) reconoce que dar posibilidades de elección y de control a los alumnos dentro del contexto de definición, selección, planificación y evaluación de las tareas y los aprendizajes genera oportunidades de tomar responsabilidad y autorregular el aprendizaje y el conocimiento. En este sentido, Paoloni (2010) concuerda con Pintrinch y Schunk (1996), que los profesores democráticos son capaces de brindar a los estudiantes un grado medio de autonomía y de control en sus procesos de aprendizaje, promoviendo la participación en la toma de decisiones y la asunción progresiva de responsabilidad.

De acuerdo con lo expresado, la propuesta de tareas en el marco del modelo de Gardner y de las características explicitadas no sólo permite concretar la idea de perspectivismo formulada por Bruner (1997), si no además se muestra como un medio para atender intereses diversos relacionados con la idiosincrasia del aprendizaje y la cognición. Además de considerar la diversidad de contextos en los que el aprendizaje puede concurrir y de hecho así sucede, y el reconocimiento de entornos naturales como fuente de conocimiento.

Por otro lado, un modelo de evaluación coherente con la propuesta de Gardner se hace presente en propuestas auténticas. Sobre éste aspectos los criterios asumidos tienen importantes aplicaciones prácticas y consecuencias para los aprendizajes. Ames (1983) considera que si el éxito queda definido en referencia a un criterio de competitividad definido por la mejor performance entre los alumnos del aula, entonces se favorecerá la comparación social. En cambio, si la evaluación se define en términos de auténtica, se entiende que es un proceso que brinda información para ser usada en la toma de decisiones educativas, para ofrecer retroalimentación a los estudiantes respecto a sus 
progresos, fortalezas y debilidades e incluso para juzgar la planificación curricular e introducir modificaciones (NCME $\left.{ }^{4}, 1990\right)$.

Al respecto, Díaz Barriga (2005), retomando los aportes de Herman, Aschbacher y Winters (1992), entiende que las prácticas de evaluación deben demandar que los aprendices resuelvan activamente tareas complejas y auténticas, haciendo uso de sus conocimientos previos, aprendizajes recientes y habilidades relevantes para la solución de problemas reales y cotidianos. Asimismo, se enfatiza que la evaluación auténtica centrada en el desempeño busca evaluar lo que se hace, así como identificar la relación de coherencia entre lo conceptual y lo procedural y entender cómo ocurre el desempeño en un contexto determinado.

En el marco formulado, se apunta a una evaluación formativa que se realice a lo largo del proceso enseñanza y aprendizaje, teniendo como objetivo fundamental regular, de manera interactiva, todo lo concerniente a la práctica pedagógica (Trillo Alonso, 2005). En este sentido, es una herramienta que le permite al docente regular la estructura, organización y desarrollo de la clase y las tareas académicas. De igual modo, se trata de un proceso de auto-evaluación que realiza el alumno, a través del cual desarrolla habilidades de autorregulación y de reflexión sobre el aprendizaje.

De hecho, la línea de trabajo sobre evaluación auténtica considera que la misma transcurre antes, durante y luego de proceso instructivo (NCME, 1990). Respecto a las actividades de evaluación que tiene lugar antes de la instrucción, se específica comprender los intereses, habilidades y capacidades de los estudiantes; comprender las motivaciones de los estudiantes hacia temas particulares; delimitar y articular las metas que se pretender lograr y planificar el proceso de enseñanza-aprendizaje conforme a la información recolectada.

\footnotetext{
${ }^{4}$ National Council on Measurement in Education, http://www.ncme.org/
} 
En relación con las actividades que tienen lugar durante el proceso se específica: el monitoreo del progreso de los estudiantes a las metas educativas acordadas; la identificación de las competencias adquiridas y las dificultades presentadas en la experiencia y las tareas propuestas; el ajuste del modelo en función de lo observado; la propuesta de instancias de feedback para avanzar en el proceso y motivar a los estudiantes. Y finalmente, luego del proceso instructivo, las actividades se encaminan a describir en qué medida cada estudiantes ha alcanzado los objetivos propuestos a mediano y largo plazo; la comunicación de las fortalezas y debilidades sobre los resultados de las evaluaciones; el análisis de la información reunida antes y durante la instrucción; la evaluación del proceso instructivo, los materiales y actividades utilizadas y los cambios a proponer.

De acuerdo con lo expuesto, Darling-Hammond, Ancess y Falk (1995) retomando a Wigging (1989) proponen cuatro características que definen a una verdadera evaluación auténtica. En cuanto a la primera característica, se considera que representan el desempeño real del campo en cuestión. Los estudiantes escriben para una audiencia real más que brindar respuestas a preguntas sobre un texto o cumplir con una prueba de ortografía, los alumnos conducen experimentos científicos, en vez de memorizar hechos de la ciencia. En este sentido, las tareas son contextualizadas y plantean desafíos intelectuales complejos que llevan al alumno a investigar y emplear el conocimiento en tareas poco estructuradas que requieren del desarrollo y uso de habilidades metacognitivas. A la vez, son tareas que dan lugar a distintos estilos intelectuales e intereses, que sirven como fuente de desarrollo de competencias e identificación de fortalezas.

Una segunda característica refiere a los criterios de evaluación. Estos permiten valorar los aspectos esenciales en distintos niveles, no centrándose en normas rígidas orientadas en una única respuesta. Asimismo, los criterios se acuerdan y expresan abiertamente y se conocen de antemano. Una tercera característica está relacionada con el papel de la 
autoevaluación, que pasa a ser parte fundamental del planteo de las evaluaciones auténticas, en tanto se busca ayudar a los estudiantes para que desarrollen la capacidad de evaluar sus trabajos al contrastarlos con estándares públicos, revisarlos, modificarlos y redirigirlos en función de sus progresos. Por último, se considera la importancia de exhibir sus trabajos al público, con doble intensión: 1- formular lo aprendido de tal forma que otros lo comprendan y 2- promover oportunidades para que otras comunidades de aprendizaje tengan acceso a la labor realizada.

En síntesis, tareas y evaluaciones, son dos elementos claves, a nuestro entender, para construir contextos educativos inteligentes desde una perspectiva psico-educativa que considere no sólo al sujetos en situación de aprendizaje, si no también al contexto educativo en general. Crear entornos de aprendizaje y enseñanza bajo el marco formulado ayuda a entender mejor las capacidades de los estudiantes, a desarrollar habilidades relevantes para la vida, a construir comprensiones más profundas de los contenidos y a generar ámbitos promotores de motivación y de compromiso.

\section{Conclusiones}

En este artículo hemos realizado una descripción de los contextos educativos inteligentes. En primera instancia aludimos a cómo y dónde ocurre cognición y aprendizaje desde una perspectiva idiosincrásica, distribuida, situada, social y contextual, rescatando la construcción de las habilidades mediadas por la experiencia y los entornos de aprendizajes.

En segundo instancia, se trabajó desde un enfoque psico-educativo con el propósito de comprender los límites y las posibilidades que se abren al superarlos cuando se comprende la importancia del contexto en el aprendizaje y se valora las múltiples capacidades de los sujetos. En este sentido, la idea de perspectivamos, contextos, 
comunidades de aprendizaje e innovaciones simbólicas son de fundamental relevancia si nos dirigimos a pensar en contextos inteligentes.

Finalmente, con el objetivo de formular alternativas prácticas, definimos en torno a las tareas académicas y las evaluaciones características y criterios que se enuncian desde las consideraciones teóricas de la psicología educacional y del enfoque socio-constructivista del aprendizaje para atender a la creación de contextos educativos inteligentes.

En suma, el trabajo ofrece algunas sendas a transitar en la consecución de entornos educativos más permisivos, flexibles y abiertos a las inteligencias de los sujetos, a configuraciones que apuesten a la motivación y al compromiso hacia los aprendizajes. Nuestro aporte se orienta a poner en práctica los principios desarrollados por la psicología de la educación para moldear contextos de enseñanza y aprendizaje que respondan, por un lado a la diversidad de formas de comprender el mundo y por otro lado a observar la riqueza de las experiencias en los contextos naturales para aprender y desarrollar habilidades. Para ello se delimitaron lineamientos generales para pensar en aulas que estén en sintonía con los aprendizajes que ocurren a diario de múltiples formas en interacción con vastos recursos y entornos...salir del aula, hacer uso de las TIC, atreverse a llevar una obra de arte al aula, invitar a profesionales, escuchar música son algunas de las tantas formas de crear contextos educativos inteligentes.

\section{Bibliografía}

Alexander, P. (2006). Psychology in Learning and Instruction. New Jersey: Pearson Education.

American Federación of Teachers, Nacional Council on Measurement in Education NCME, National Education Association (1990). Standards for teacher competences in educational assessment of students. Washington, DC: American Psychological Association. 
Ames, C. (1992). Classroom: Goals, structures, and student motivation. Journal of Educational Psychology, 84 (3), 261-271.

Ames, C. \& Archer, J. (1988). Achievement goals in the classroom: students'learning strategies and motivation processes. Journal of Educational Psychology, 80 (3), 260-267.

Boix Mansilla, V. (2004). Abriendo Puertas a las artes, la mente y más allá. México: Conaculta.

Bruner, J. (1997). La educación puerta de la cultura. Madrid: Visor.

Díaz Barriga, F. (2005). Enseñanza Situada: vínculo entre la escuela y la vida. México: McGraw Hill.

Falk y Dierking (2000). Learning from Museum. Visitor Experiences and the Making of Meaning. Walnut Creek, CA: AltaMira Press.

Gardner, H. (1983). Frames of mind: The theory of multiples intelligences. New York: Basic Book.

Gardner, H. (2006). Multiple Intelligences. New Horizons. New York: Basic Book.

Kelly, D. \& Tangney, B. (2006) Adapting to intelligence porfile in an adaptive educational system. Interacting with Computers, 18, 385-409.

Molina Ruiz, E (2005) Creación y desarrollo de comunidades de aprendizaje: hacia la mejora educativa. Revista de Educación, 337, 235-250.

Paoloni, P. (2010) El Contexto de aprendizaje. Implicancias en el estudio de la motivación académica. En Rinaudo, C. y Donolo, D. (Comp.) Estudios sobre motivación: enfoques, resultados, lineamientos para acciones futuras (pp. 35-46) Río Cuarto: Editorial de la Universidad Nacional de Río Cuarto. 
Pea, R. (1993). Prácticas de inteligencia distribuida y diseños para la educación. En Salomón, G. (edit.). Cogniciones distribuidas. Consideraciones psicológicas y educativas (pp. 75-125). Buenos Aires: Amorrortu.

Perkins, D. (1995). La escuela inteligente. Del adiestramiento de la memoria a la educación de la mente. Barcelona: Gedisa.

Rinaudo, C. \& Donolo, D. (2000) Casandra y la educación. La universidad como contexto de aprendizaje. En Guerci de Siufi, B. (Comp.) Pensando la Universidad. (pp. 105150). Jujuy: Editorial UNJU y Red de Editoriales de Universidades Nacionales.

Rosenholtz, S. \& Wilson, B. (1980). The effect of classroom structure on shared perceptions of ability. American Educational Research Journal, 17 (1), 75-82.

Trillo Alonso, F. (2005). Competencias dicentes y evaluación auténtica: ¿Falla el protagonismo? Colección de Cuadernos de actualización para pensar la Enseñanza Universitario, № 3. UNRC. 